\title{
Research and application of Hubei electric power company anti- electricity stealing laboratory
}

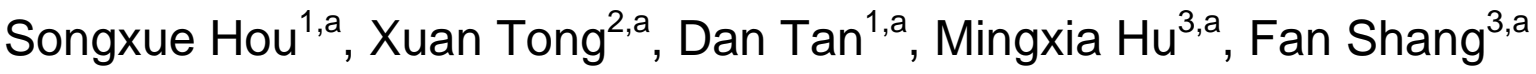 \\ ${ }^{1}$ Technical training center of Hubei electric power company, Wuhan, 430079, China \\ ${ }^{2}$ Hubei electric power company, Wuhan 430071, China \\ ${ }^{3}$ Customer service center of Hubei electric power company, Wuhan 430071, China \\ aemail: 909030532@qq.com, bemail:5392441@qq.com, cemail:806242187@qq.com
}

Keywords: Anti-electricity Stealing; Training; Case Analysis; Simulation

\begin{abstract}
In order to effectively combat the act of electricity stealing which had long plagued power supply enterprises, it is essential to train power consumption inspectors on that act of electricity stealing in theory and form. This paper first illustrates the importance and technical difficulties of anti-electricity stealing training. On that basis, the construction principle, functional features and technical indexes of anti-electricity stealing laboratory are expounded. Finally, the application of the achievement is introduced. The laboratory introduced in this paper is in the domestic leading level of anti-electricity stealing training, which has great impact on strengthening the technical level and promoting the quality of staff. In addition, anti-electricity stealing training increases the profits of power enterprises and ensures the continuous process of electric power energy reproduction work.
\end{abstract}

\section{Introduction}

With the rapid development of smart grid, the measures of electricity stealing is becoming more and more intelligent and concealment. Technical training of staff must be strengthened and quality of staff should be improved, only in this way the act of electricity stealing can be combated and the profits of power enterprises can be increased. The technology training of anti-electricity stealing laboratory in Hubei electric power company comprises skill training and simulation training. The training is dominated by monitoring and analyzing the data from information acquisition system with the goal of practical marketing electricity inspection. The laboratory is planned and constructed from aspects of case presentation, principle analysis, field operation and technical research of anti-electricity stealing. It solves the disconnection between traditional training and reality and anti-electricity stealing skill training usually lags behind the measures of electricity stealing. The simulation system uses the typical measurement methods and $10 \mathrm{kV}$ multi-user utility lines to simulate various electricity stealing measures for anti-electricity stealing training. The platform architecture design is in the leading position on anti-electricity stealing training in the country.

\section{The construction principle and features of anti-electricity stealing laboratory}

The anti-electricity stealing laboratory comprises skill training and simulation training. Its main function is realizing data sharing based on data collection simulation system and operational training of anti-electricity stealing skills, constituting the data linkage training platform of antielectricity stealing.

\subsection{Construction principle}

The construction of laboratory embodies the principle of "practical real-based, appropriately advanced"; the training equipment of anti-electricity stealing and power information simulation system embody the principle of "complementary universal, data linkage"; the training equipment embodies the principle of "simulation realistic, emulation operation". 


\section{(1) The simulation principle}

The simulated training uses the electric energy measurement device which is synchronous with the on-site, thus providing a real environment of anti-electricity stealing. Through teacher's computer or installing actual electricity stealing measurement device, teacher can simulate different types of electricity stealing. Trainee will analyze the certain type of electricity stealing teacher set so that determine the method of electricity stealing and respond accordingly according to the data from information acquisition system and on-site practical inspection results.

(2) The communication compatibility principle of electric power information acquisition system

Laboratory system is the application system based on electric power acquisition system simulation. This training system requires all the communication interface and protocol must be compatible with information acquisition system and load control system:

1) All simulation data and the feature set of simulation training system must be able to be uploaded to electricity information acquisition and monitoring system, and the abnormal using of electricity is found by acquisition system;

2) There is a data interaction between acquisition device of every subsystem and host station of "electricity information acquisition simulation training system". All used equipment and communication protocol are compatible with electricity information acquisition simulation training system, and can communicate with the main control software of laboratory.

(3) The principle of practicality

The simulation training system adopts the equipment type of hardware settings which are consistent with the practical work to the greatest extent. Only Trainee who have passed the examination and inspected various typical electricity stealing measures proficiently are allowed to work.

(4) The principle of reliability

The software design must lay emphasis on the principle of reliability. Modular design, redundancy data processing design should not affect the platform's working ability. Server and system design should ensure that the system's working stability and the promptness of simulation data will not be affected by factors, such as long running time of system, increase in item bank of skill identification, growth of system data and change of system functions and so on.

(5) The principle of innovation

The simulation training system of anti-electricity stealing adopts the electric energy measurement device which is synchronous with the on-site. Accordingly, it will strengthen trainee's visual impression so that they can master the principle and methods of anti-electricity stealing in the process of operating by themselves.

1) Set up posts' relevant workstation with the function of training and practice.

2) Set up posts' relevant workstation with the function of skill identification and examination.

3) Set up projection screen to increase the visibility of the practical teaching.

4) The simulation of the terminal data acquisition.

5) The simulation of data interface and data transmission.

6) Set up the skill identification and skill identification examination item bank.

\subsection{Function features}

The laboratory of anti-electricity stealing is divided into four parts, new technology research and case demonstration area, theory teaching and acquisition analysis area, anti-electricity stealing simulation training area and practical operation area.

(1) New technology research and case demonstration area

New technology research area is used to do research on case of electricity stealing, new technology and methods of anti-electricity stealing and methods of obtaining evidence on the platform of measuring cabinet, meters, transformers and other metering device. Demonstrating the tools of anti-electricity stealing at home and abroad and develop effective anti-electricity stealing device using research platform.

Case demonstration area is composed of static display screen and dynamic display screen. It has 
functions of both teaching and memorizing, trainee can quickly learn the rationale and measures of electricity stealing to improve the level of anti-electricity stealing.

(2) Theory teaching and acquisition analysis area

Training courses can make trainee gain deep insight into relevant theoretical foundation knowledge on marketing metrics with the combination of PPT and teaching demonstration on display screen. Through analyzing the real data of the on-site, using remote positioning inspection method, the high-loss lines and suspicious electricity stealing users can be filtrated. In addition, regular on-site actions of anti-electricity stealing are taken to optimize the methods and working process.

(3) Anti-electricity stealing simulation training area

The simulation training area with skill training network system is mainly composed of the teaching screens. Each group's screen is designed in the manner of high voltage power supply high voltage measurement, high voltage power supply low voltage measurement and low voltage power supply low voltage measurement. Two sets touch screen and fifty-six locations of anti-electricity stealing are set for each teaching screen. Forty people can be hold in this area and there are desks, projection, audio and other multimedia training equipment.

1) The principle of measurement and the process of electricity stealing, such as shunting, undervoltage, misphase, strong magnetic field interference, remote control can be studied by simulation training system.

2) Instruction mode and practice mode can be set respectively. Trainers can explain the variation of measurement loop parameters of each link under instruction mode; trainee can simulate operation of electricity stealing according to the training platform on the screen under practical mode and decide whether it is abnormal using of electricity or not.

3) The type and location of electricity stealing can be analyzed according to the data on the screen.

4) Trainers can instruct the platform of electricity stealing via broadcast to realize the examination function of teaching system.

(4) Anti-electricity stealing practical operation area

The practical operation area is designed based on typical measurement of $10 \mathrm{kV}$ distributed line. The electricity stealing locations are hidden in the measurement loop and the information is transmitted to the teaching host.

1) The practical operation system simulates four branch users of $10 \mathrm{kV}$ line of substation, including outdoor high voltage power supply high voltage measurement anti-electricity stealing simulation operation platform(branch 1), outdoor high voltage power supply low voltage measurement anti-electricity stealing simulation operation platform(branch 2), indoor high voltage power supply high voltage measurement anti-electricity stealing simulation operation platform(branch 3) and low voltage power supply low voltage measurement anti-electricity stealing simulation operation platform(branch 4). The increase of line loss can be simulated to achieve the goal of examining and competing on anti-electricity stealing skills.

2) Determine the area and measure of electricity stealing by analyzing the high loss branch. Thereby, trainee can master specific determining methods and investigating progress of electricity stealing.

3) The competition of anti-electricity stealing can be achieved via training operation system in various modes, such as group match, optional extracting examination and random extracting examination and so on.

\subsection{Technological Analysis}

Anti-electricity stealing laboratory can meet the needs of anti-electricity stealing training under the background of intelligent power grid. In order to overcome the related difficulties, key technology is innovated in the process of design construction.

(1) Primary current measurement

To make sure the training of inspectors can reflect the user's real electricity consumption situation, electrifiable high voltage wireless detection device can be used to collect the user's 
primary load. The device's technical requirements are shown in table 1.

\begin{tabular}{|c|c|}
\hline Technical requirements & Index \\
\hline Current load measurement range & $\begin{array}{c}0-200-300 \mathrm{~A} \\
\end{array}$ \\
\hline Voltage grade & $0.40 \sim 35 \mathrm{kV}$ \\
\hline Applicative wire diameter & (No measurement of dead zone) \\
\hline Accuracy of measurement & $100 \mathrm{~m}$ \\
\hline Wireless transmission distance & $-40^{\circ} \mathrm{C} \sim+65^{\circ} \mathrm{C}$ \\
\hline The environment temperature & Electrifiable loading and unloading \\
\hline Installation method & w
\end{tabular}

Tab.1.Technical index of high voltage wireless detection device

(2) Data record and recall

In order to test inspection personnel's training effect and realize the informatization of inspecting, data recording device can be used to learn how to use data recording device to auxiliary forensics through man-machine interface system. The device's technical requirements are shown in table 2.

\begin{tabular}{|c|c|}
\hline Technical requirements & Index \\
\hline Communication Protocol & Standard specification of State Grid（DL/T645-1997-2007） and SG186 system interface \\
\hline \multirow{4}{*}{ Communication interface } & GPRS interface \\
\hline & RS485 data interface \\
\hline & Radio frequency communication interface \\
\hline & USB maintenance interface \\
\hline The data collection & $\begin{array}{l}\text { Receiving data from high voltage wireless current transformer and user's measurement } \\
\text { table }\end{array}$ \\
\hline $\begin{array}{l}\text { Strong magnetic } \\
\text { monitoring }\end{array}$ & When strong magnetic field disturbs, the device automatically send alarm information. \\
\hline \multirow{2}{*}{$\begin{array}{l}\text { Electromagnetic } \\
\text { compatibility }\end{array}$} & $\begin{array}{c}\text { Radio frequency electromagnetic field radiation immunity-GB/T 17626.3-2006 Harsh level } \\
\text { is Level III. }\end{array}$ \\
\hline & Power frequency magnetic field immunity-GB/T 17626.8-2006 Harsh level is Level IV. \\
\hline
\end{tabular}

\section{(3) Load box}

Load box is a kind of equipment that can simulate the variation of resistive load, capacitive load and inductive load in daily life. It's mainly used for field calibration, test equipment, etc. Technology of load box device is shown in table 3.

\begin{tabular}{|c|c|}
\hline Technical requirements & Index \\
\hline Measuring range of phase voltage & 0 - $300 \mathrm{~V}$, precision is $\pm 0.2 \%$ 、 the voltage resolution is $0.1 \mathrm{~V}$ \\
\hline Measuring range of current & $0-5 \mathrm{~A}$, precision is $\pm 0.2 \%$ 、 the current resolution is $0.1 \mathrm{~A}$ \\
\hline Measuring range of active power & 0 - $3.3 \mathrm{kw}$, precision is $\pm 0.5 \%$ 、 the power resolution is $0.01 \mathrm{kw}$ \\
\hline The minimum step amplitude & $\begin{array}{l}\text { Resistive load, capacitive load and inductive load are } \\
\text { continuously adjustable,the minimum step amplitude is } 15^{\circ}\end{array}$ \\
\hline Measuring range of reactive power & $\begin{array}{c}0-3.3 \mathrm{kw} \text {, precision is } \pm 0.5 \% \text { 、 } \\
\text { the power resolution is } 0.01 \mathrm{kw}\end{array}$ \\
\hline Cooling way & Forced air cooling fan \\
\hline Connection mode & $\begin{array}{l}\text { Lower wiring patter } \\
\text { Three phase four wire system Y type connection, there are A、B、C、 } \\
\text { N four connection mouth. } \\
\text { Single phase can connect two wire system from } \\
\text { A-N, B-N and C-N. }\end{array}$ \\
\hline Working power supply & AC $220 \mathrm{~V} / 50 \mathrm{HZ}$ \\
\hline Environmental conditions & $\begin{array}{c}\text { Saving environment temperature }-25^{\circ} \sim 70^{\circ} \\
\text { Working environment temperature }-10^{\circ} \sim 40^{\circ} \\
\text { relative humidity } 5 \% \sim 95 \% \\
\text { barometric pressure } 80 \mathrm{kPa} \sim 110 \mathrm{kPa} \text { (Under } 2 \mathrm{~km} \text { altitude) }\end{array}$ \\
\hline
\end{tabular}

Tab.3.Technical index of load box device

(4) Anti-electricity stealing simulation training screen

Anti-electricity stealing skills training platform can help trainers analyze the theory of electricity 
stealing and train anti-electricity stealing skills on several form of measurement such as measuring in high voltage side and supplying in high voltage side, supplying in high voltage side and measuring in low voltage side and supplying in low voltage side and measuring in low voltage side. It can also demonstrate the operation training and make the students master the theory knowledge of electricity stealing and train anti-electricity stealing skills. The technical requirements of antielectricity stealing simulation training screens are shown in table 4.

\begin{tabular}{|c|c|}
\hline Technical requirements & Index \\
\hline Voltage grade & AC 380V $\pm 15 \%$ AC $220 \mathrm{~V} \pm 15 \%$. frequency $50 \pm 0.5 \mathrm{~Hz}$ \\
\hline Screen structure & $\begin{array}{l}\text { Three relatively independent screens combine as one set of platform,a large } \\
\text { analog metering system diagram is printed on it and each metering system } \\
\text { can form a system independently. The platform support touch operation } \\
\text { mode and computer display. }\end{array}$ \\
\hline Operator mode & Local operation and simulation operation two modes \\
\hline Communication methods & GPRS data transmission and RS232 directly connected \\
\hline $\begin{array}{c}\text { Electricity stealing point } \\
\text { setting }\end{array}$ & Invisible design \\
\hline Intelligent load & 3KVA/0.1K Intelligent adjustable \\
\hline Boundary dimension & One set of platform 2100mm(height)*750mm(width )*2400mm(depth) \\
\hline
\end{tabular}

Tab.4.Technical index of anti-electricity stealing simulation training screens

(5) Anti-electricity stealing simulation practical operation screen

Anti-electricity stealing simulation practical operation screen highly simulates typical measurement design of $10 \mathrm{kV}$ line. It can realize a variety of simulation of metering fault and electricity stealing by the load controlling system. It can also simulate electricity stealing in user and the change of actual line loss and position the electricity stealing area and specific way of electricity stealing, then it can combine the electricity information acquisition system and real electricity stealing environment to simulate the power utilization environment. The technical requirements of anti-electricity stealing simulation practical operation screen are shown in table 5.

\begin{tabular}{|c|c|}
\hline Technical requirements & Index \\
\hline Working power supply & $\begin{array}{c}\mathrm{AC} 380 \mathrm{~V} \pm 15 \% 、 \mathrm{AC} 220 \mathrm{~V} \pm 15 \% \text { 、 } \\
\text { frequency } 50 \pm 0.5 \mathrm{~Hz}\end{array}$ \\
\hline Intelligent load & 3KVA $/ 0.1 \mathrm{~K}$ Intelligent adjustable \\
\hline Electricity stealing point setting & Invisible design \\
\hline Operator schema & Local operation and simulation operation two modes \\
\hline Boundary dimension & $\begin{array}{c}\text { One simulation user } \\
\text { 2450mm(height)*1020mm(width)*2500mm(depth ) }\end{array}$ \\
\hline Three-phase load cases & $\begin{array}{c}\text { Resistive load, capacitive load, } \\
\text { adjustable inductive load }\end{array}$ \\
\hline
\end{tabular}

Tab.5.Technical index of anti-electricity stealing simulation practical operation screen

\section{The advancement of anti-electricity stealing laboratory}

\subsection{Current situation of anti-electricity stealing training}

The research of anti-electricity stealing develops slowly abroad, there is only electric energy meter and no work on specific anti-electricity stealing demo system has been carried out. At home, companies and research institutions has paid more and more attention to anti-electricity stealing and has carried out some research on related technology and equipment. At present, many antielectricity stealing products including anti-electricity stealing electric energy meter and antielectricity stealing demo training device have been put in the market. However, the existing various anti-electricity stealing demo training device is still stay in simulating the electricity stealing caused by wrong wiring and can not simulate the electricity stealing caused by additional device or 
changing the internal parameters of the electric energy meter. So these existing device can not training the worker about the electricity stealing manners such as shunting electricity stealing, remote control electricity stealing, high frequency interference electricity stealing and changing the sampling resistor in electric energy meter. Therefore, they can not meet the needs of anti-electricity stealing training under the background of power grid intelligence.

3.2 The advantage of anti-electricity stealing laboratory

The anti-electricity stealing laboratory is divided into new anti-electricity stealing technology and case display area, theoretical teaching and collection and analysis area, anti-electricity stealing simulation training area and anti-electricity stealing real operation area four parts.

(1) Innovations of the new anti-electricity stealing technology and case demonstration area

1) Dynamically displaying typical electricity stealing (controlled).

2) Typical destructive or unrepeatable electricity stealing can be displayed through threedimensional animation.

3) Displaying anti-electricity stealing achievement in Hubei province.

4) Dynamically connecting local anti-electricity stealing cases and remaining updating material periodically.

(2) Innovations of the theoretical teaching and acquisition analysis area

It provides trainee the SG186 system and electricity consumption information acquisition system which are the same system used on-site. The data is on-site data and simulation operation training data which are updated periodically so that trainee will be familiar to their work. Through the analysis of the real on-site data, the use of the remote location inspection method could find lines with high loss and suspicious electricity stealing users. Then the on-site electricity stealing action could be organized termly and the working method and procedure could also be optimized.

(3) Innovations of the anti-electricity stealing simulation training area

Researching the electricity stealing process and summarizing anti-electricity stealing manners through setting typical various electricity stealing methods including shunting, undervoltage, misphase, strong magnetic field interference, remote control and primary side lapping. After setting aforementioned electricity stealing manners, the electricity stealing way and the stealing position could be established and various electricity stealing manners could also be understood.

(4) Innovations of the anti-electricity stealing practical operation area

Trainee can observe actual electricity consumption and combine theory and reality though reappearing actual electricity stealing by simulating on-site metering devices configuration mode, including measuring in high voltage side and supplying in high voltage side, supplying in high voltage side and measuring in low voltage side and supplying in low voltage side and measuring in low voltage side.

\section{Promotion prospects}

Promoting the prospects is of great significance in power grid and technical development. It is mainly reflected in the promotion in control ability of power supply enterprise, running stability and energy conservation and loss reduction of distribution network.

(1) Improving control ability of power supply enterprise.

The rapid development of economic construction in our country makes the electricity consumption and electricity sales increasing rapidly year by year, which follows with the increase of electricity stealing events. Some enterprise whose major cost factor is electricity, such as some iron and steel smelting, cement, chemical industry, hotel and so on, have more latent force on electricity stealing. As a result, the research on anti-electricity stealing technology improves the control ability of power supply enterprise.

(2) Maintaining stable running of distribution network

The electricity stealing behavior or using electricity violating a treaty of some enterprises and individuals will cause adverse effect to the stable running of the distribution network. Therefore, the research on anti-electricity stealing technology is significant and can maintain the stable running of 
distribution network.

(3) Energy conservation and loss reduction.

The research on anti-electricity stealing technology can improve the control ability of enterprises and maintain stable operation of distribution network. Then the energy conservation and loss reduction will achieve significant economic benefits.

In order to improve the technological level and personnel quality of the electricity inspector, electricity metering personnel, meter installing and electricity connecting personnel, rural electric power marketing worker and electric consumption personnel in the whole province, Hubei electric power company marketing department plan to set up anti-electricity stealing knowledge base all around the province and municipal or county-level company can consider to establish small and medium-sized anti-electricity stealing technology training researching platform by combining the facilities resource and using the advanced technology of anti-electricity stealing laboratory.

\section{Application}

At present, the electricity consumption information acquisition system and high and low voltage measurement anti-electricity stealing technology laboratory set up by HuBei electric power company technical training center have been put into use. Though conducting hierarchical training of multiple skills on power consumption inspectors, electricity metering personnel, meter installing and electricity connecting personnel, rural electric power marketing workers, so that multi-level training function, “teaching, learning, practice and competition”, have been implemented. The level of the electric power marketing training ground has been improved cooperated with the smart grid construction.

In the anti-electricity stealing training segment, the system will be combined with anti-electricity stealing practical application training, measuring device principle analysis and research on typical electricity stealing methods. In order to cooperate with the need of fighting against electricity stealing, loss reduction and energy saving and maintaining the normal order of electricity consumption, anti-electricity stealing training on related electricity inspectors should be conducted and high-tech anti-electricity stealing tools should be carried out.

In the simulation, practical operation and examination segment, the practical operation of antielectricity stealing methods and measuring equipment simulation will be taught and examined. The seamless connection between training and practical operation can be realized through simulating various electricity stealing situations to train practical anti-electricity stealing methods using computer technology, simulation technology and virtual load technology. Then the training process can be equipped with multi-level function including teaching, learning, training and racing, it can realise both teaching anti-electricity stealing theory and displaying various electricity stealing methods.

In the combination of theory and practice segment, SG186 system and existed electricity consumption information acquisition system will be combined to set up intelligent remote energy consumption monitoring and line loss management analysis platform of entire network. The platform can connect the anti-electricity stealing equipment of on-site typical users with remote access and analyse electricity stealing methods in real time. By breaking through the traditional way of training, tracking the electricity inspection of trainee, analysing actual electricity consumption information of users and intensively explaining related knowledge about measuring system such as the type of measuring equipment, parameters, fault type, connection mode, work flow and the process procedure of abnormal state.

\section{Conclusion}

The anti-electricity stealing laboratory built by Hubei electric power company can simulate various electricity stealing measures and anti-electricity stealing methods, and then conduct related anti-electricity stealing training. The structure design of the platform has taken the lead in the antielectricity stealing field in domestic. 
The establishment of anti-electricity stealing laboratory can meet the requirements of antielectricity stealing training under the background of smart grid. Though the training, the technical level of the staff could be strengthened and the personnel quality could be improved and various electricity stealing could be understood in theory and form. In addition, the economic benefits of the electric power enterprises could be increased and the reproduction work of electrical energy can be undertaken continuously.

\section{References}

[1] LI Xiangjun.Discussion on the application of electric information acquisition system in antistealing[J]. Science and technology information, 2014, (10).

[2] SU Hongru, YANG Hua, LUO Xiaoxue.Study on the problems and measures of anti-stealing work in the power supply enterprises[J].The innovation of science and technology guide newspaper, 2014, (11).

[3] HUO Xiao. Development and Application of Intelligent Electricity Anti-stealing System Based on Power Consumption Information Acquisition[D]. North China Electric Power University, 2014.

[4] CHENG Chao, ZHANG Hanjing, JING Zhimin, CHEN Ming, JIAO Lei, YANG Lixin. Study on the anti-electricity stealing based on outlier algorithm and the electricity information acquisition system[J]. Power System Control and Protection, 2015, 43(17).

[5] SUN Xiaoqing. Research and Design of Intelligent Domestic Security System Based on the Wireless Communication[D]. Hunan University, 2012.

[6] JIANG Jiaolian, TANG Lizhong, BAI Bing.The design of GPRS microseismic monitoring alarm message system[J]. Industry and Mine Automation, 2010, (5).

[7] JING Ping, ZHOU Fei, SONG Jieying, GUO Jianbo.The design and Simulation of unified power flow controller with modular structure[J].Power System Technology, 2013, (2) 